\title{
Is Treatment of Segond Fracture Necessary with Combined Anterior Cruciate Ligament Reconstruction : Letter to the editor
}

Etienne Cavaignac*,MD (1), Adnan Saithna, MD PhD (2), Edoardo Monaco, MD PhD (3),

Camilo P Helito,MD PhD (4), Matthew Daggett, MD (5), Nicolas Reina, MD PhD (1),

Bertrand Sonnery-cottet, MD (6)

(1): Department of Orthopedic Surgery and Trauma, Hôpital Pierre Paul Riquet, Toulouse,

France

(2): School of Science and Technology, Nottingham Trent University, Nottingham, England

(3) : University of Rome La Sapienza, Sant'Andrea Hospital, Rome, Italy

(4): IOT HCFMUSP, Sao Paulo, Brazil

(5): Kansas City University

(6): Centre Orthopedique Santy, Lyon, France

\section{*Corresponding author:}

Etienne Cavaignac, MD

Department of Orthopedic Surgery

Hopital Pierre Paul Riquet, CHU Toulouse

Rue Jean Dausset

3105 Toulouse, France

Telephone number: +33.607 .342 .595$

Office telephone number: +33.561 .322 .712$

Fax number: +33.561 .322 .232$

Dear Editor, 
We read the article entitled "Is Treatment of Segond Fracture Necessary with Combined Anterior Cruciate Ligament Reconstruction" by Melugin et al. with great interest. ${ }^{12}$ This article focuses on the management of Segond fractures in patients undergoing ACL reconstruction.

The primary challenge resides in detecting the said lesion. Segond fractures are typically identified on standard knee radiographs. ${ }^{5}$ However, using only radiographs underestimates the number of patients with a bony injury of the anterolateral aspect of the knee. A Segond fracture can be more reliably detected with other imaging modalities. ${ }^{41}$ Plain radiographs have a lower spatial resolution than MRI and especially ultrasonography (US). ${ }^{13}$ This means US is more likely to detect small bone lesions. Various studies on this topic have shown that US detects a larger number of Segond fractures than MRI, with MRI being better than plain radiographs. Klos et al. reported a much higher rate of detection (30\%) when using US. ${ }^{11}$ Cavaignac et al. found a large difference in the detection rate of Segond lesions based on plain radiographs (3.3\%), MRI (13\%) and US (50\%). ${ }^{1}$

Ultrasonography makes it possible to detect a certain number of Segond fractures that are not fractures by definition, but actually unicortical lesions over the ALL's tibial insertion. ${ }^{2}$ A hematoma will often be present at the ALL/bone junction. ${ }^{2}$ This is not a fracture by definition, but a bone lesion. ${ }^{3}$ In our opinion, it is preferable to use the term "Segond lesion" instead of "Segond fracture". The latter should be used only when a fracture is visible on radiographs, while the former can be detected with other imaging modalities. ${ }^{4} 71$

This left us wondering whether the control group in the Melugin et al. ${ }^{12}$ study had Segond lesions not seen on plain radiographs, which would result in an analysis bias that no longer makes the groups comparable. Some patients in the control group may have belonged in the Segond fracture group.

While it can be said that a Segond lesion corresponds to an ALL lesion, ${ }^{5}$ not every ALL lesion is a Segond lesion. ${ }^{8}{ }^{9}$ The indirect question asked by Melugin et al. ${ }^{12}$ directly leads to the treatment of two injuries: ACL and anterolateral structures. The problem is that their control group likely contained patients with an anterolateral ligament injury that was missed because they were not revealed by the presence of a radiographically detectable Segond fracture. In our opinion, this is a major confounding factor in the study. Each patient underwent an MRI examination. The anterolateral region of the knee could have been analyzed with MRI, instead of solely with radiographs.

The plausibility of a result is an important element for validating the methodology of a study. Melugin et al. compared their clinical outcomes to the findings published by SonneryCottet et al. after ACL and ALL reconstruction. ${ }^{14,15}$ In their eyes, their clinical outcomes were comparable to published results. We believe it would be relevant for Melugin et al. to disclose the prevalence of Segond lesions found on plain radiographs and MRI, especially since the patients have already undergone an MRI. If the prevalence of Segond fractures is very low, then an analysis bias may be present due to their poor ability to diagnose Segond fractures.

Melugin et al $^{12}$ reported their analysis of the healing of Segond fractures. Can effective healing be seen on plain radiographs? We believe that plain radiographs are not sufficient to draw conclusions about fracture healing. Due to bone superimposition, bone fragments may 
appear to be continuous with the anterolateral portion of the tibia even when the bones have not yet healed. Only 3D imaging (CT scan or MRI) or imaging focused on this area (US) can determine whether this type of injury has healed.

The lack of difference between the study and control groups can be due to several factors: 1- there is actually no difference, 2- the tools used to measure the difference were not powerful enough to detect it, 3- the groups being compared only differ in the studied variable, 4- the minimal clinically important difference (MCID) used was not determined from a comparable population. We believe the last three factors can explain the findings of Melugin et al. ${ }^{12}$ We identified several sources of bias: analysis bias (underestimation of the number of Segond lesions), selection bias (potential Segond lesions in the control group), interpretation bias (20\% lost to follow-up; distribution of BPTB / hamstring graft not comparable between groups). To answer the question: "Is Treatment of Segond Fracture Necessary with Combined Anterior Cruciate Ligament Reconstruction?" a study with a population of ACL-injured patients with Segond fracture that compares a group undergoing ACL reconstruction only to a group undergoing a combined ACL / anterolateral procedure would be more appropriate in our opinion. Furthermore, the MCID of 11.5 cited, was based on a study of patients with a mean age of 40.5 years (range 12.5 to 81.3 ) and the most frequent diagnosis in that group was osteoarthritis. ${ }^{10}$ To our knowledge there are no studies determining the MCID of the IKDC score in young patients with ACL injury. However, in contrast, other authors have reported that the alternative metric of minimal detectable difference is as low as 6.5. ${ }^{6}$ This suggests caution in assuming that the failure of the statistically significant difference to reach the threshold of 11.5 IKDC points truly reflects non-importance.

In the paper's discussion, Melugin et al. single out the extra-articular lateral tenodesis indication for ALL reconstruction. This is certainly a significant problem that requires a consensus approach. We believe there are two types of indications for ALL reconstruction: preventative and curative. It has been shown that adding lateral tenodesis to ACL reconstruction reduces the risk of ACL re-tear. ${ }^{14}$ Thus it appears this additional procedure is beneficial in populations with a high re-tear risk, no matter the condition of the ALL. While this at-risk population has not yet been precisely defined, some candidates are high-level athletes, young adults, and those who participate in pivoting or contact sports. We also believe that there are curative indications related to damaged structures, i.e. ALL reconstruction when this structure is visibly damaged It will be difficult to evaluate the best indications with a clinical study; in fact, no study design can truly answer this question. An expert consensus could be a starting point...

To conclude, we believe that the term "Segond fracture" should be reserved for bony avulsions seen on plain radiographs. The term "Segond lesion" is more appropriate when (1) other imaging modalities can be used to detect damage to the anterolateral structures of the knee, and (2) there is a unicortical lesion or bone avulsion of the ALL. Moreover, we want to stress that a Segond lesion is not the only type of anterolateral lesion that can occur. The study by Melugin et al ${ }^{12}$ did well to shine the spotlight on the treatment of Segond lesions when they occur with an ACL tear; however, no conclusion about treatment can be drawn based on their methodology. The indications for additional procedures during ACL reconstruction must still be defined. 


\section{References:}

1. Cavaignac E, Faruch M, Wytrykowski K, et al. Ultrasonographic Evaluation of Anterolateral Ligament Injuries: Correlation With Magnetic Resonance Imaging and Pivot-Shift Testing. Arthroscopy. 2017;33(7):1384-1390.

2. Cavaignac E, Laumond G, Reina N, et al. How to Test the Anterolateral Ligament With Ultrasound. Arthroscopy techniques. 2018.

3. Cavaignac E, Wytrykowski K, Murgier J, Reina N, Chiron P, Faruch M. Regarding "Editorial Commentary: Ultrasound Barely Beats Magnetic Resonance Imaging in Knee Anterolateral Ligament Evaluation ... But Does This Change the Treatment of the Anterior Cruciate Ligament-Deficient Knee?". Arthroscopy. 2017;33(11):19181919.

4. Cavaignac E, Wytrykowski K, Reina N, et al. Ultrasonographic Identification of the Anterolateral Ligament of the Knee. Arthroscopy. 2016;32(1):120-126.

5. Claes S, Luyckx T, Vereecke E, Bellemans J. The Segond fracture: a bony injury of the anterolateral ligament of the knee. Arthroscopy. 2014;30(11):1475-1482.

6. Collins NJ, Misra D, Felson DT, Crossley KM, Roos EM. Measures of knee function: International Knee Documentation Committee (IKDC) Subjective Knee Evaluation Form, Knee Injury and Osteoarthritis Outcome Score (KOOS), Knee Injury and Osteoarthritis Outcome Score Physical Function Short Form (KOOS-PS), Knee Outcome Survey Activities of Daily Living Scale (KOS-ADL), Lysholm Knee Scoring Scale, Oxford Knee Score (OKS), Western Ontario and McMaster Universities Osteoarthritis Index (WOMAC), Activity Rating Scale (ARS), and Tegner Activity Score (TAS). Arthritis Care Res (Hoboken). 2011;63 Suppl 11:S208228.

7. Faruch Bilfeld M, Cavaignac E, Wytrykowski K, et al. Anterolateral ligament injuries in knees with an anterior cruciate ligament tear: Contribution of ultrasonography and MRI. Eur Radiol. 2018;28(1):58-65.

8. Ferretti A, Monaco E, Fabbri M, Maestri B, De Carli A. Prevalence and Classification of Injuries of Anterolateral Complex in Acute Anterior Cruciate Ligament Tears. Arthroscopy. 2017;33(1):147-154.

9. Helito CP, Helito PVP, Costa HP, Demange MK, Bordalo-Rodrigues M. Assessment of the Anterolateral Ligament of the Knee by Magnetic Resonance Imaging in Acute Injuries of the Anterior Cruciate Ligament. Arthroscopy. 2017;33(1):140-146.

10. Irrgang JJ, Anderson AF, Boland AL, et al. Development and validation of the international knee documentation committee subjective knee form. Am J Sports Med. 2001;29(5):600-613.

11. Klos B, Scholtes M, Konijnenberg S. High prevalence of all complex Segond avulsion using ultrasound imaging. Knee Surg Sports Traumatol Arthrosc. 2017;25(4):13311338.

12. Melugin HP, Johnson NR, Wu IT, Levy BA, Stuart MJ, Krych AJ. Is Treatment of Segond Fracture Necessary With Combined Anterior Cruciate Ligament Reconstruction? Am J Sports Med. 2017:363546517745280.

13. Nazarian LN. The top 10 reasons musculoskeletal sonography is an important complementary or alternative technique to MRI. AJR Am J Roentgenol. 2008;190(6):1621-1626.

14. Sonnery-Cottet B, Barbosa NC, Vieira TD, Saithna A. Clinical outcomes of extraarticular tenodesis/anterolateral reconstruction in the ACL injured knee. Knee Surg Sports Traumatol Arthrosc. 2017. 
15. Sonnery-Cottet B, Saithna A, Cavalier M, et al. Anterolateral Ligament Reconstruction Is Associated With Significantly Reduced ACL Graft Rupture Rates at a Minimum Follow-up of 2 Years: A Prospective Comparative Study of 502 Patients From the SANTI Study Group. Am J Sports Med. 2017;45(7):1547-1557. 